\title{
Pressure and pressure gradient of laser-induced emergent stress waves using 0.07-mm-thick black rubber target
}

\author{
Koji Aizawa ${ }^{1, *}$ and Yoshiaki Tokunaga ${ }^{2}$ \\ ${ }^{1}$ Center for Electric, Optic and Energy Applications, Kanazawa Institute of Technology, \\ 7-1 Ohgigaoka, Nonoichi, 921-8501 Japan \\ ${ }^{2}$ Optoelectronic Device System Research \& Development Center, Kanazawa Institute of Technology, \\ 7-1 Ohgigaoka, Nonoichi, 921-8501 Japan
}

(Received 26 June 2014, Accepted for publication 30 June 2014)

Keywords: Emergent stress wave, Laser, Black rubber, Acoustic signature, Pressure, Pressure gradient PACS number: 43.35.Ud [doi:10.1250/ast.36.155]

\section{Introduction}

The transfer methods of foreign substances, such as drugs and DNA, into cells using photomechanical waves have been of interest in cell biology because of the possibility of biomedical applications. The successful transfection of plasmid DNA into mammalian cells using a laser-induced stress wave (LISW) has been demonstrated [1,2]. An LISW, a compressive and strong stress pulse with sub-microsecond duration, can be produced by irradiating a nanosecond laser pulse to an optically opaque material (laser target) covered with a transparent sheet, owing to pressure confinement. Since the LISW is generated by complex integration of nonlinear phenomena such as ablation, heat plasma, detonation, and shock waves, the authors call such a stress wave a laserinduced emergent stress wave (LIESW) [3]. In the transfer method using the LIESW, an abrupt pressure variation in the culture medium is applied to the targeted cells. The peak pressure and pressure gradient increase the permeability of the cell membrane and transfer efficiency of foreign substances and hence are significant parameters $[2,4]$.

In previous studies, a 0.5 -mm-thick black rubber sheet was typically used as the laser target $[1,2]$. The black rubber possesses extraordinary properties of not only as optical absorber but also an acoustical material with impedance matching to tissue, and also functions as a shock absorber owing to its viscoelasticity. To reduce the influence of acoustic power absorption and frequency dispersion during the propagation of the LIESW, a target thinner than $0.5 \mathrm{~mm}$ should be examined. On the other hand, it has been found that the rubber components are ablated from the surface to within the depth range of approximately $30 \mu \mathrm{m}$ [5]. A target thicker than about $0.05 \mathrm{~mm}$ would be needed to maintain the confined pressure. For these reasons, we experimentally investigated the effect of a thin black rubber target of $0.07 \mathrm{~mm}$ thickness by evaluating the temporal pressure profile (acoustic signature).

\section{Experimental procedure}

Ethylene propylene diene modification (EPDM) and polyethylene terephthalate (PET) are chosen as the black

\footnotetext{
*e-mail: aizawa@neptune.kanazawa-it.ac.jp
}

rubber target and transparent material, respectively. An EPDM/PET structure (area: $10 \times 10 \mathrm{~mm}^{2}$ ), that is, 0.07 mm-thick EPDM (Daiko-Rubber, EPDM0.07mm) adhered to 1-mm-thick PET (Acrysunday, PG-1) using epoxy resin as adhesive, was fabricated in this experiment.

To generate the LIESW, a Q-switch Nd:YAG (Spectra Physics, LAB-130) was used as the laser source, in which the wavelength of laser light was $532 \mathrm{~nm}$ for second-harmonic generation. The measured maximum output pulse energy in single-shot operation was approximately $0.14 \mathrm{~J} /$ pulse. A laser pulse with a $10 \mathrm{~ns}$ duration was focused on the EPDM surface through the PET sheet of the EPDM/PET structure, using a convex lens (focal length: $200 \mathrm{~mm}$ ). The irradiated laser intensity was changed by using a neutral density filter. The laser fluence, defined as the laser energy divided by the ablated area, was varied from 0.23 to $1.25 \mathrm{~J} / \mathrm{cm}^{2}$. The diameter of the ablated area was approximately either 2.3 or $4.4 \mathrm{~mm}$. Figures 1(a) and 1(b) show the setups for measuring the acoustic signature on the back of the target and in water, respectively. In the setup shown in Fig. 1(a), 110- $\mu$ m-thick poly(vinylidene difluoride) (PVDF, Measurement Specialties, distributed by Tokyo Sensor) was used as the transducer. The PVDF transducer stacked on 10-mm-thick natural rubber (NR) as the absorber was placed on an EPDM/PET structure, and then the interfaces between them were joined using a reflection-coefficient-matched gel (Fukuda, UF cleargel) in order to reduce the reflection of acoustic waves at their interfaces. In Fig. 1(b), a glass base dish (Iwaki, glass thickness: $0.1 \mathrm{~mm}$ ) filled with pure water was fixed on the EPDM/PET structure. The acoustic signature in water was measured using a hydrophone probe (Muller Instruments, sensitive diameter: $<0.5 \mathrm{~mm})$. The probe tip was placed approximately $1 \mathrm{~mm}$ away from the bottom of the dish at the target center. These acoustic signatures were recorded using a sampling digital oscilloscope (LeCroy, LC684DXL), in which the time interval between sample points was fixed at $0.5 \mathrm{~ns}$.

\section{Results and discussion}

Figures 2(a), 2(b), and 2(c) show the typical temporal profiles of the sensing signal from the PVDF transducer, the pressure measured with a hydrophone probe, and the pressure 

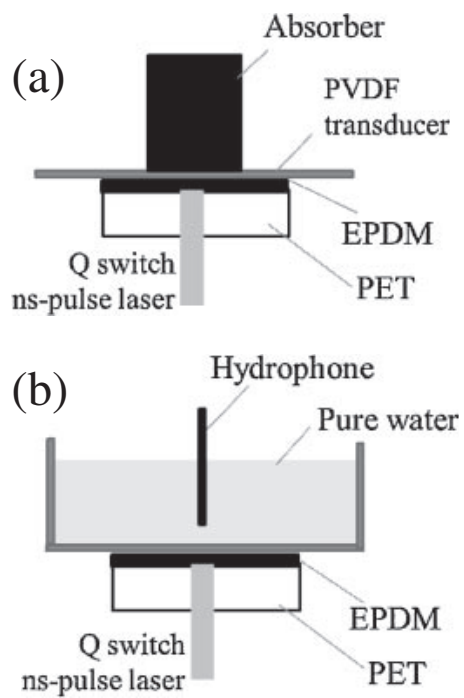

Fig. 1 Illustrations of measurement setups for measuring acoustic signature using (a) a PVDF transducer and (b) a hydrophone probe.

gradient, respectively, when the laser fluence was $0.91 \mathrm{~J} / \mathrm{cm}^{2}$. To calculate the pressure from the output voltage of the hydrophone probe, the sensitivity of approximately 72.6 $\mathrm{MPa} / \mathrm{V}$ was used. The pressure gradient was defined as the pressure difference divided by the interval time of $5 \mathrm{~ns}$. As shown in Figs. 2(a) and 2(b), the strong positive (compressive) pressure was predominant. The peak pressure in water was approximately $30 \mathrm{MPa}$ under the above condition. The LIESW through water propagates as a plane wave within the distance of up to $5 \mathrm{~mm}$ from the target surface [6]. The full width at half maximum (approximately $180 \mathrm{~ns}$ ) in water was three times larger than that of the transducer signal owing to the dispersion effect of water. The temporal profile after passing through the peak pressure was changed by the multiple reflection of the LIESW. The maximum pressure gradient, calculated from Fig. 2(b), became approximately $1.5 \mathrm{MPa} / \mathrm{ns}$, as shown in Fig. 2(c). Although the negative pressure shown in Fig. 2(b) seems to be a considerable value owing to bubble formation, the pressure gradient after passing through the peak pressure is much smaller than the maximum value. Since the targeted cells are physically influenced by pressure in the culture medium, the peak pressure and maximum pressure gradient in water are particularly investigated hereafter.

To clarify the thickness effect of the laser target, a 2mm-thick NR (Best Sound Labo, NR2mm) was used for comparison with EPDM0.07mm. The sound velocity and the carbon black content of NR were almost the same as those of EPDM. Figures 3(a) and 3(b) show the laser fluence dependences of the peak pressure and maximum pressure gradient, respectively. It was found from Fig. 3(a) that the measured peak pressure values correspond to the laser fluence to the 0.5 th power when the laser fluence is higher than approximately $0.6 \mathrm{~J} / \mathrm{cm}^{2}$. As an example, the peak pressure when using of EPDM $0.07 \mathrm{~mm}$ became approximately three times higher than that when using in NR2 $\mathrm{mm}$, when the laser fluence of $1 \mathrm{~J} / \mathrm{cm}^{2}$
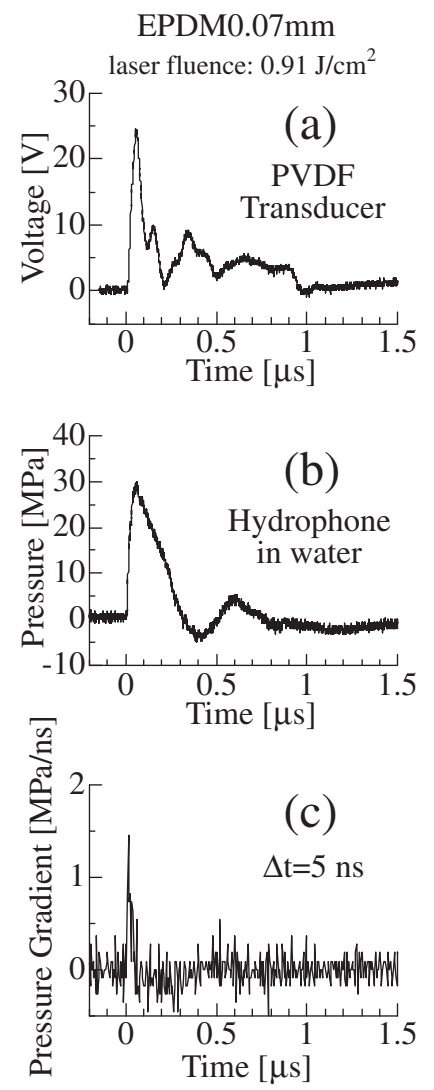

Fig. 2 Typical temporal profiles of (a) voltage signal from the PVDF transducer, (b) pressure measured with the hydrophone probe, and (c) pressure gradient. The pressure gradient was defined as the pressure difference divided by the time interval $(\Delta \mathrm{t})$ of $5 \mathrm{~ns}$. EPDM0.07mm was used as the laser target. The laser fluence was kept at $0.91 \mathrm{~J} / \mathrm{cm}^{2}$.

was applied to the laser target. The decrease in peak pressure in the thicker target suggests power-law absorption in the loss medium. As shown in Fig. 3(b), the maximum pressure gradient when using of EPDM0.07mm became approximately ten times larger than that when using of NR2mm at the laser fluence of $1 \mathrm{~J} / \mathrm{cm}^{2}$. Since the propagation time in EPDM0.07mm is shorter than that in NR2 $\mathrm{mm}$, these results suggest a change in the wave shape during travel through the dispersive medium.

When the applied pulse energy is high enough to generate the shock wave in a confined geometry, the shock pressure $P$ is described by the following relation [7]:

$$
P=a \sqrt{\frac{Z}{\tau} F}
$$

where $a, Z, \tau$, and $F$ are a constant, the shock impedance, the pulse duration, and the laser fluence, respectively. Since the measured peak pressure in the laser fluence range higher than $0.6 \mathrm{~J} / \mathrm{cm}^{2}$ obeys this relation regardless of target thickness, the LIESW originates from the shock wave formed at the interface between the black rubber and PET.

Figure 4 shows the relationship between the peak pressure and maximum pressure gradient. It is revealed that the 


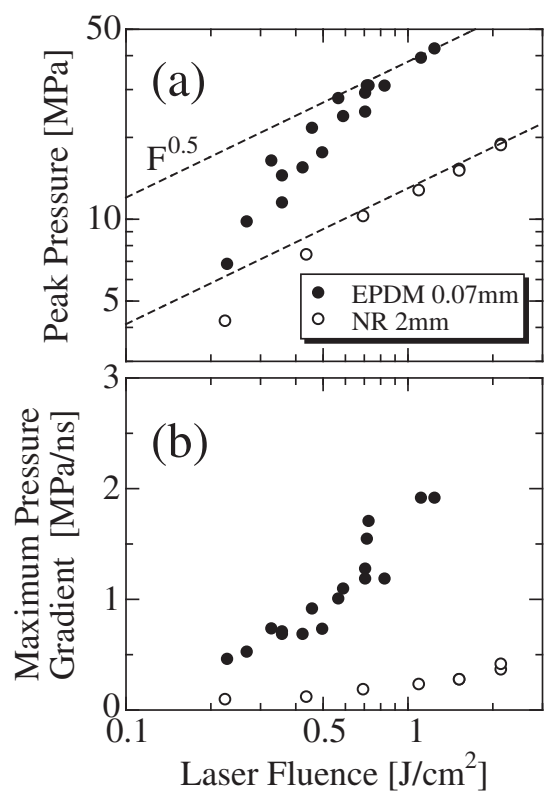

Fig. 3 (a) Peak pressure and (b) maximum pressure gradient variations as functions of laser fluence. The solid and open circles indicate the results for EPDM $0.07 \mathrm{~mm}$ and NR $2 \mathrm{~mm}$, respectively. The dashed lines show the dependence on the laser fluence $(F)$ to the power of 0.5 .

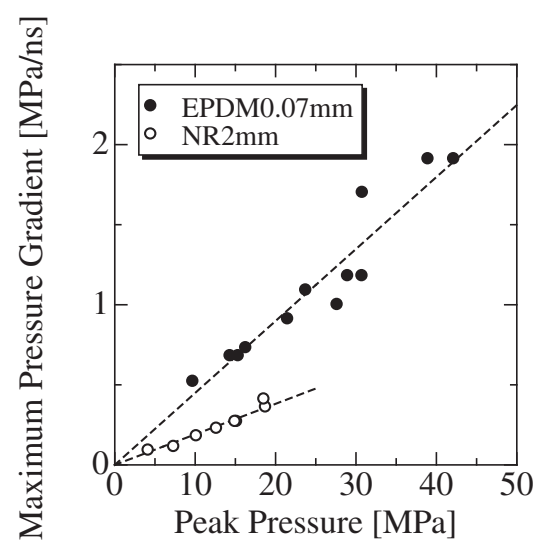

Fig. 4 Relationship between peak pressure and maximum pressure gradient. The results obtained using (a) EPDM $0.07 \mathrm{~mm}$ and (b) NR $2 \mathrm{~mm}$ are indicated by the solid and open circles, respectively. The dashed lines show the least-squares fitting to the measured data.

pressure gradient is generally proportional to the peak pressure. The result in EPDM0.07mm shows a proportionality approximately 2.5 times larger than that in NR2mm. In other words, a pressure gradient 2.5 times larger than when using NR2mm was obtained at the same peak pressure.

In this study, the peak pressure and maximum pressure gradient at the laser fluence of $1.25 \mathrm{~J} / \mathrm{cm}^{2}$ became approximately $42 \mathrm{MPa}$ and $1.9 \mathrm{MPa} / \mathrm{ns}$, respectively. These values seem to be sufficient for achieving the transfer of plasmid
DNA into several mammalian cells [8]. As an effect of the EDPM0.07mm target on biological application, a transfer efficiency larger than $80 \%$ of that of dextran into HeLa cells was introduced by the authors [9].

\section{Conclusion}

The temporal profiles of stress waves generated by the irradiation of a high-energy laser pulse with a $10 \mathrm{~ns}$ duration onto a $0.07-\mathrm{mm}$-thick black rubber target covered with a 1-mm-thick transparent sheet were investigated. The peak pressure and maximum pressure gradient of these waves increased in comparison with those when using a 2-mm-thick black rubber target, and thus were sufficient for the transfer of foreign substances into mammalian cells. Consequently, 0.07mm-thick black rubber is one of promising candidates for the laser target of an LIESW device in biomedical applications.

\section{Acknowledgment}

The authors acknowledge the useful advice of Professor M. Kogi of Kanazawa Institute of Technology, and wish also to thank Mr. A. Tominaga and Mr. S. Orisaka for their technical assistance. This work was partly supported by a Grant-in-Aid from the Japan Society for the Promotion of Science (Nos. 23656275 and 24560384).

\section{References}

[1] M. Ogura, S. Sato, K. Nakanishi, M. Uenoyama, T. Kiyozumi, D. Saitoh, T. Ikeda, H. Ashida and M. Obara, "In vivo targeted gene transfer in skin by the use of laser-induced stress waves," Lasers Surg. Med., 34, 242-248 (2004).

[2] M. Terakawa, M. Ogura, S. Sato, H. Wakisaka, H. Ashida, M. Uenoyama, Y. Masaki and M. Obara, "Gene transfer into mammalian cells by use of a nanosecond pulsed laser-induced stress wave," Opt. Lett., 29, 1227-1229 (2004).

[3] Y. Tokunaga, M. Nishiwaki and K. Aizawa, "Comprehensive research on emergence of impulsive stress wave using a nanosecond pulse laser," Proc. Symp. Ultrason. Electron., 33, 93-94 (2012).

[4] A. G. Doukas and S. Lee, "Photomechanical drug delivery," Proc. SPIE, 3916, 188-197 (2000).

[5] S. Yoshida, T. Makino, Y. Tokunaga and K. Aizawa, "Report on emergent stress wave formed by Q-switched ns-pulse lase, II," IEICE Tech. Rep., US2012-15, pp. 11-16 (2012) (in Japanese).

[6] T. Ando, S. Sato, S. Takano, H. Ashida and M. Obara, "Propagation characteristics of laser-induced stress wave in deep tissue for gene transfer," Appl. Surf. Sci., 255, 9898-9901 (2009).

[7] R. Fabbro, J. Fournier, P. Ballard, D. Devaux and J. Virmont, "Physical study of laser-produced plasma in confined geometry,” J. Appl. Phys., 68, 775-784 (1990).

[8] M. Terakawa, S. Sato, H. Ashida, K. Aizawa, M. Uenoyama, Y. Masaki and M. Obara, "In vitro gene transfer to mammalian cells by the use of laser-induced stress waves: effects of stress wave parameters, ambient temperature, and cell type," $J$. Biomed. Opt., 11, 014026 (2006).

[9] S. Nishimura, M. Kogi, T. Yanagisawa, S. Orisaka, K. Aizawa and Y. Tokunaga, "Effect of laser-induced emergent stress wave on cell adhesion," Proc. Symp. Ultrason. Electron., 34, 345-346 (2013). 